\title{
Dual-mode quasi-logarithmic quantizer with embedded G.711 codec
}

\author{
Bojan Denić*, Zoran Perić*, Vladimir Despotović**, \\ Nikola Vučić*, Predrag Petrović ${ }^{* * *}$
}

\begin{abstract}
The G.711 codec has been accepted as a standard for high quality coding in many applications. A dual-mode quantizer, which combines the nonlinear logarithmic quantizer for restricted input signals and G.711 quantizer for unrestricted input signals is proposed in this paper. The parameters of the proposed quantizer are optimized, where the minimal distortion is used as the criterion. It is shown that the optimized version of the proposed quantizer provides 5.4 dB higher SQNR (Signal to Quantization Noise Ratio) compared to G.711 quantizer, or equivalently it performs savings in the bit rate of approximately $0.9 \mathrm{bit} / \mathrm{sample}$ for the same signal quality. Although the complexity is slightly increased, we believe that due to the superior performance it can be successfully implemented for high-quality quantization.

K e y w or d s: scalar quantization, G.711 standard, Laplacian source, signal to noise ratio
\end{abstract}

\section{Introduction}

Scalar quantization is an important area of signal processing that offers simple and fast algorithms appropriate for real time applications that require small encoding delay [1-4]. It refers to the quantization scheme where the source signal is quantized one sample at time. In general, it falls into two categories, uniform and non-uniform quantization.

Although it is the simplest, the uniform scalar quantization has been extensively investigated in the literature [1-5]. High resolution uniform scalar quantization has been considered in [5], where the asymptotic analysis of the optimal fixed-rate uniform scalar quantization was performed.

Non-uniform quantization refers to using the nonequal spacing of quantization levels. Its major advantages compared to the uniform quantization are the increased dynamic range and the possibility to design a quantizer tailored to the specific input statistics, thus providing the superior performance [3]. A direct approach for locating the best non-uniform quantizer, if the probability density function (PDF) of the source signal is known, is the determination of decision boundaries and reconstruction levels that minimize the mean squared quantization error using the iterative Lloyd-Max's algorithm [1-4]. The other approach is using the companding, where the input is first transformed through a nonlinear compressor function, which is further quantized using the uniform quantizer, and finally restored in expander using the inverse nonlinear function. The compressor and the expander constitute the compander. The most widely used are logarithmic and optimal companders. Specifically, the piecewise linear approximation to the logarithmic or optimal compression function has gained a great interest due to the easier hardware and software implementation. For example, in ITU-T G.711 standard [6] the piecewise linear approximation to $\mu$-law or A-law logarithmic characteristic is implemented. The linearization of optimal compression function has been reported in [7].

ITU-T G.711 codec is extensively used in many contemporary systems. It is an inevitable part in public switched telephone network (PSTN) and voice over internet protocol (VoIP) [8,9].

Although companding is dominantly used in speech coding, it was also applied in other fields. For example, in [10] it was employed for the purpose of reducing the long-term evolution (LTE) networks traffic. Beside companding, another possibility to increase the signal quality and reduce the complexity of design and realization is to exploit the log-polar uniform quantization [11].

It is known that for a symmetric input distribution with infinite support (eg Laplacian, Gaussian), the quantizer range is divided into granular and overload region. The designed dynamic range of the quantizer defines the granular region, while the range of values outside this dynamic range is called the overload region. The quality of the quantized signal is influenced by the width of the granular region and if the input signal exceeds it, the clipping occurs, which introduces the error known as the overload distortion. If the granular region is decreased, the space between its output values is also decreased, leading to smaller granular distortion, but at the same time increasing the overload distortion. Hence, the quantizer design requires determining a balance between the granular and the overload distortion [12].

* Deparment of Telecommunications, Faculty of Electronic Engineering, Univeersity of Niš, A. Medvedeva 14, 18000 Niš, Serbia, bojan.denic@elfak.rs, zoran.peric@elfak.ni.ac.rs ** Technical Faculty in Bor, University of Belgrade, Vojske Jugoslavije 12, 19210 Bor, Serbia, vdespotovic@ tfbor.bg.ac.rs, *** IRITEL AD Beograd, Batajnički put 23, 11080 Belgrade, Serbia presa@iritel.com 
When the bounds of the quantizer support region are infinite we consider the case of unrestricted quantizer. Restricting the bounds to finite values defines the restricted quantizer. The combination of restricted and unrestricted quantization was recently proposed in designed for Laplacian sources and in for Gaussian sources.

The input signal is processed frame-by-frame and the adaptive restricted companding quantizer was used whenever the amplitude range for the particular frame falls within its support region, while for other frames the unrestricted quantizer was used. Using this approach, the elimination of the overload distortion of the restricted companding scalar quantizer was ensured.

In this paper we use the similar idea, but design restricted and unrestricted quantizers in different way. As opposed to [13-15], the proposed quantizer uses the embedded G.711 quantizer for unrestricted input, while the restricted quantizer is based on the logarithmic companding, both with equal number of quantization levels. In this way, the performance of G.711 quantizer can be significantly improved. The parameters of the proposed quantizer are optimized in terms of minimal distortion for a given frame length. Furthermore, an example of nonoptimal parameters selection and their influence on the performance has been considered as well. It will be shown that the small introduced complexity compared to the G.711 quantizer can be traded for the achieved enhancement.

The remainder of this paper is organized as follows: in Section 2 a description of the proposed dual-mode quasilogarithmic quantizer and its main components, the logarithmic companding and G.711 quantizer is provided.. In Section 3 we present and discuss the numerical results and finally we conclude the paper in Section 4 .

\section{Scalar companding quantization}

A scalar quantizer with $N$ levels is specified by the set of real numbers $x_{1}, x_{2}, \ldots, x_{N_{1}}$, called decision thresholds, satisfying $-\infty<x_{1}<x_{2}<\cdots<x_{N-1}<$ $x+N<\infty$ and set of numbers $y_{1}, y_{2}, \ldots, y_{N}$ called representation levels, satisfying $y_{i} \in \alpha_{i}=\left(x_{i-1}, x_{i}\right]$ for $i=1, \ldots, N$ [12]. Where $\alpha_{i}$ is denoted as the quantization cell, where $\bigcup_{i=1}^{N} \alpha_{i} \in R$ and $\alpha_{i-1} \cap \alpha_{i}=0$. The quantizer is defined as many-to-one mapping $Q: R \rightarrow$ $\left\{y_{1}, y_{2}, \ldots, y_{N}\right\}$ defined by $Q(x)=y_{i}$, when $x \in \alpha_{i}$. Additionally, for the assumed nonlinear input source, cells $\alpha_{2}, \ldots, \alpha_{N}$ form the granular region and are called granular cells, while $\alpha_{1}$ and $\alpha_{N}$ constitute an overload region and are called overload cells.

In the following subsections we describe the combined quasi-logarithmic quantizer which is composed of the restricted quantizer based on logarithmic companding and the unrestricted embedded G.711 quantizer, designed for Laplacian sources.
2.1 Restricted quantizer based on logarithmic companding

In this paper we use the non-uniform quantization based on the companding technique, mainly due to the increased design complexity of the algorithm proposed by Lloyd and Max [1]. The building blocks of each scalar compandor are the compressor, the uniform quantizer and the expander, connected in cascade [1-4], as illustrated in Fig. 1. Hence, the following steps are encountered when processing the input signal $x$ : compress the input signal $x$ by applying the compressor function $c(x)$; apply the uniform quantizer to the compressed signal $Q_{u}(c(x))$; and expand the quantized version of the compressed signal using an inverse compressor function $c^{-1}\left(Q_{u}(c(x))\right)$.

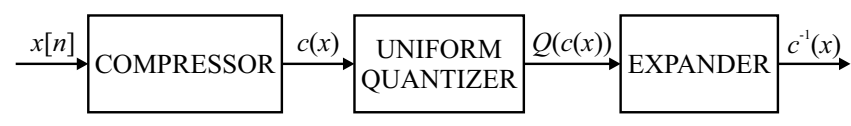

Fig. 1. The nonuniform quantization realized by scalar compounding technique

We consider a logarithmic compression function [1-4], which two variants, $\mu$-law and A-law compression function, are incorporated in extensively used G.711 standard [6].

In $\mu$-law, compressor function is given by [1-4]

$$
c(x)=\frac{x_{\max }}{\ln (1+\mu)} \ln \left(1+\frac{\mu|x|}{x_{\max }}\right) \operatorname{sgn}(x),|x| \leq x_{\max },
$$

where $\mu$ is the compression factor and $x_{\max }$ is the upper support region threshold of the $\mu$-law quantizer.

Let us define the decision thresholds $x_{u, i}$ and the representative levels $y_{u, i}$ of the uniform quantizer

$$
\begin{gathered}
x_{u, i}=-x_{\max }+\frac{2 i}{N} x_{\max },, \quad i=0, \ldots, N, \\
y_{u, i}=-x_{\max }+\frac{2 i-1}{N} x_{\max }, \quad i=1, \ldots, N .
\end{gathered}
$$

After companding, we can determine the parameters of the equivalent non-uniform quantizer (thresholds $x_{i}$ and levels $y_{i}$ ) from the conditions

$$
c\left(x_{i}\right)=x_{u, i}, \quad c\left(y_{i}\right)=y_{u, i}
$$

resulting in

$$
\begin{aligned}
& x_{i}=\frac{x_{\max }}{\mu}\left((1+\mu)^{\left.\frac{x_{u, i} \operatorname{sgn}\left(x_{u, i}\right)}{x_{\max }}-1\right) \operatorname{sgn}\left(x_{u, i}\right), i=0, \ldots, N,},\right. \\
& y_{i}=\frac{x_{\max }}{\mu}\left((1+\mu)^{\left.\frac{y_{u, i} \operatorname{sgn}\left(y_{u, i}\right)}{y_{\max }}-1\right) \operatorname{sgn}\left(y_{u, i}\right), i=0, \ldots, N .}\right.
\end{aligned}
$$

We assume, without loss of generality, that the information source that needs to be quantized is memoryless, 
zero-mean and unit variance $\left(\sigma^{2}=1\right)$ Laplacian with the probability density function $(\mathrm{PDF})[1-4]$ :

$$
p(x)=\frac{1}{\sqrt{2}} \exp (-\sqrt{2}|x|) .
$$

Since we deal with the symmetric source, the following identities will hold: $x_{i}=-x_{N+2-i}$ and $y_{i}=-y_{N+1-i}$, $i=1, \ldots, N$.

Let us introduce the mean-squared distortion as a measure of irreversible error incurred during the quantization, which consists of the granular $D_{\mathrm{g}}$ and the overload $D_{\mathrm{o}}$ component. For the $\mu$-law quantizer and unit variance case, they are given by [16]

$$
\begin{gathered}
D_{\mathrm{g}}=\frac{\ln ^{2}(1+\mu)}{3 N^{2}}\left(\frac{x_{\max }^{2}}{\mu^{2}}+\frac{2 x_{\max } \overline{|x|}}{\mu}+\overline{x^{2}}\right), \\
D_{\mathrm{o}}=2 \int_{x_{\max }}^{\infty}\left(x-y_{N}\right)^{2} p(x) \mathrm{d} x .
\end{gathered}
$$

where $\overline{|x|}$ and $\overline{x^{2}}$, for the Laplacian PDF in (7), are given as

$$
\begin{aligned}
& \overline{|x|}= \int_{-x_{\max }}^{x_{\max }} x p(x) \mathrm{d} x= \\
& \frac{1}{\sqrt{2}}\left(1-\exp \left(-\sqrt{2} x_{\max }\right)\left(1+\sqrt{2} x_{\max }\right)\right), \\
& \overline{x^{2}}=\int_{-x_{\max }}^{x_{\max }} x^{2} p(x) \mathrm{d} x= \\
& 1-\exp \left(-\sqrt{2} x_{\max }\right)\left(x_{\max }^{2}+\sqrt{2} x_{\max }+1\right) .
\end{aligned}
$$

Now, substituting (10) and (11) into (8), the granular distortion becomes

$D_{\mathrm{g}}=\frac{\ln ^{2}(1+\mu)}{3 N^{2}}\left(\frac{x_{\max }^{2}}{\mu^{2}}+\frac{\sqrt{2} x_{\max }}{\mu}\left(1-\exp \left(-\sqrt{2} x_{\max }\right) \times\right.\right.$

$\left.\left.\left(1+\sqrt{2} x_{\max }\right)\right)-\exp \left(-\sqrt{2} x_{\max }\right)\left(x_{\max }^{2}+\sqrt{2} x_{\max }+1\right)+1\right)$.

Further, by approximating $y_{N}$ with $x_{\max }$, (9) becomes

$$
D_{\mathrm{o}}=\exp \left(-\sqrt{2} x_{\max }\right) .
$$

By using (12) and (13) we obtain the following closedform expression for the total distortion $\mathrm{D}$

$$
\begin{array}{r}
D=\frac{\ln ^{2}(1+\mu)}{3 N^{2}}\left(\frac{x_{\max }^{2}}{\mu^{2}}+\frac{\sqrt{2} x_{\max }}{\mu}\left(1-\exp \left(-\sqrt{2} x_{\max }\right) \times\right.\right. \\
\left.\left.\left(1+\sqrt{2} x_{\max }\right)\right)-\exp \left(-\sqrt{2} x_{\max }\right)\left(x_{\max }^{2}+\sqrt{2} x_{\max }+1\right)+1\right) \\
+\exp \left(-\sqrt{2} x_{\max }\right) .
\end{array}
$$

SQNR is used for performance evaluation of the quantizer [1-4]:

$$
\mathrm{SQNR}=10 \log _{10} \frac{1}{D}
$$

\subsection{Unrestricted embedded G.711 quantizer}

ITU-T G.711 standard [6] defines the companded (non-uniform) quantization algorithm, where the logarithmic compression function is approximated by the piecewise linear functions. It encodes signal according to either $\mu$ law or $A$-law compressor function [6]. Its support region $\left[-x_{\max }^{\mathrm{G} .711}, x_{\max }^{\mathrm{G} .711}\right]$, is partitioned into $2 L=16$ unequal segments (each consecutive segment is twice as large as previous), where each segment contains $\mathrm{m}$ uniform cells. Furthermore, it is symmetric, meaning that 8 segments are positive and 8 symmetrically distributed segments are negative. The segments width denoted by $\Delta_{i}^{\text {G.711 }}$ can be determined as

$$
\Delta_{i}^{\mathrm{G} .711}=2^{i} \frac{x_{\max }^{\mathrm{G} .711}}{255 m}, \quad i=0,1, \ldots, L-1,
$$

where $x_{\max }^{\mathrm{G} .711}$ is the upper support region of the G.711 quantizer determined as in [17]:

$$
x_{\max }^{\mathrm{G} .711}=\frac{1}{\sqrt{2}} \log \frac{3 \mu N^{2}}{\log (\mu+1)} .
$$

where $N$ is the number of quantization levels. Note that in case the $\mu$-law is selected, the G.711 standard recommends the use of $\mu=255$ and $N=256$ [6].

The borders between the segments $x_{i}^{\mathrm{G} .711}$ are

$$
x_{i}^{\mathrm{G} .711}=\frac{\left(2^{i}-1\right) x_{\max }^{\mathrm{G} .711}}{255}, \quad i=0,1, \ldots, L,
$$

while the cells borders $x_{i j}^{\mathrm{G} .711}$ and the representative levels $y_{i j}^{\mathrm{G} .711}$ in the $i$-th segment $(i=0,1, \ldots, L-1)$ are

$$
\begin{aligned}
& x_{i j}^{\mathrm{G} .711}=x_{i}^{G .711}+j \Delta_{i}^{\mathrm{G} .711}, \quad j=0,1, \ldots, m, \\
& y_{i j}^{\mathrm{G} .711}=x_{i}^{\mathrm{G} .711}+\frac{(2 j-1)}{2} \Delta_{i}^{\mathrm{G} .711}, j=1, \ldots, m .
\end{aligned}
$$

In case of the Laplacian PDF given with (7), granular and overload distortion of the G.711 quantizer can be expressed in the form [1]

$$
\begin{array}{r}
D_{\mathrm{g}}^{\mathrm{G} .711}=\sum_{i=0}^{L-1} \frac{\left(\Delta_{i}^{\mathrm{G} .711}\right)^{2}}{12}\left(\exp \left(-\sqrt{2} x_{i}^{\mathrm{G} .711}\right)-\right. \\
\left.\exp \left(-\sqrt{2} x_{i+1}^{\mathrm{G} .711}\right)\right),
\end{array}
$$

$$
\begin{aligned}
& D_{\mathrm{o}}^{\mathrm{G} .711}= \\
& \quad \exp \left(-\sqrt{2} x_{\max }^{\mathrm{G} .711}\right)\left(\left(x_{\max }^{\mathrm{G} .711}-y_{L-1, m}^{\mathrm{G} .711}+\frac{1}{\sqrt{2}}\right)^{2}+\frac{1}{2}\right)
\end{aligned}
$$

where $y_{L-1, m}^{\mathrm{G} .711}$ is the representative level of the last cell in the last segment, obtained according to (20).

SQNR is defined as

$$
\mathrm{SQNR}^{\mathrm{G} .711}=10 \log _{10} \frac{1}{D_{\mathrm{g}}^{\mathrm{G} .711}+D_{\mathrm{o}}^{\mathrm{G} .711}}
$$




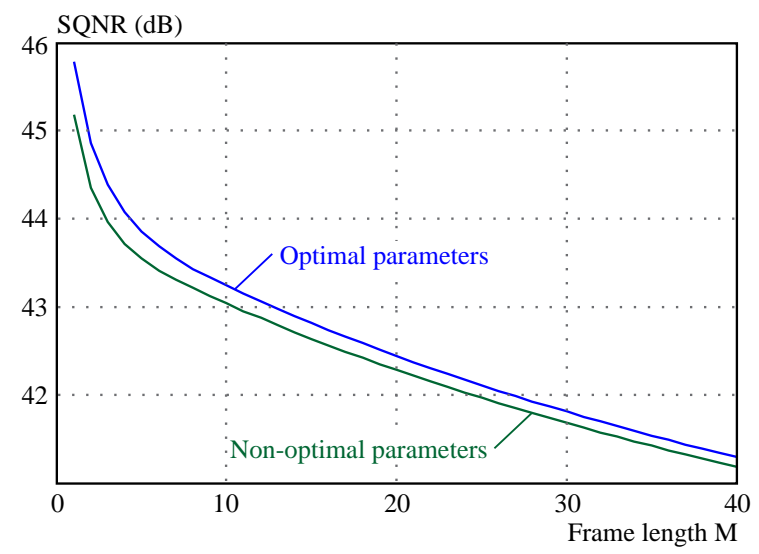

Fig. 2. SQNR dependence on the frame length $M$

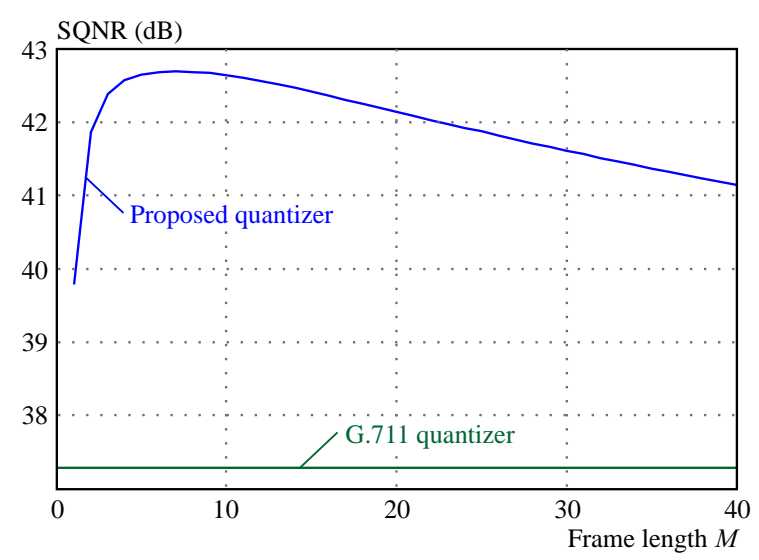

Fig. 3. Selection of the optimal frame length for $R=8 \mathrm{bit} / \mathrm{sample}$

\subsection{Description of the dual-mode quasi-logarithmic quantizer}

In this section, the dual-mode quasi-logarithmic quantizer is introduced. It is based on the approach presented in [13-15], involving the restricted logarithmic quantizer (denoted by $\log \mathrm{Q}$, see Section 2.1) and the unrestricted quantizer based on the G.711 standard (see Section 2.2), both designed for Laplacian source with zero-mean and unit variance and equal number of quantization levels $N$. Particularly, the restricted $\log \mathrm{Q}$ having a smaller support region is designed to quantize the input signals bounded by amplitude. On the other hand, the G.711 quantizer with wider support region is exploited for unbounded input signals (unrestricted quantizer). In this way we are able to quantize any input signal, bounded or unbounded by amplitude.

The input signal is divided into frames and processed frame-by-frame. For each frame a switching rule is defined to choose one of the available quantizers. The restricted $\log \mathrm{Q}$ is selected whenever the maximal absolute signal amplitude of the current frame is within its support region. Observe that in this case only the granular distortion of $\log \mathrm{Q}$ should be taken into account (overload distortion is equal to zero). In case the maximal absolute amplitude falls outside the support region of the restricted $\log \mathrm{Q}$, the quantizer based on G.711 standard is selected.
Total distortion incurred by the proposed combined quasi-logarithmic quantizer can be expressed as $[13,14]$

$$
D_{t}=P^{\mathrm{r}} D_{\mathrm{g}}^{\mathrm{r}}+\left(1-P^{\mathrm{r}}\right) D^{\mathrm{G} .711} .
$$

where $D_{\mathrm{g}}^{\mathrm{r}}$ is the granular distortion of the restricted $\log \mathrm{Q}$ (see (14)), and $D^{\mathrm{G} .711}$ denotes the sum of granular and overload distortion of the G.711 quantizer given by (21) and $(22)$, respectively. $P^{\mathrm{r}}$ denotes the probability of selection of the restricted $\log \mathrm{Q}[13,14]$

$$
P^{\mathrm{r}}=\left(2 \int_{0}^{x_{\max }^{\mathrm{r}}} p(x) \mathrm{d} x\right)^{M}=\left(1-\exp \left(-\sqrt{2} x_{\max }^{\mathrm{r}}\right)\right)^{M} .
$$

where $M$ is the frame size and $x_{\max }^{\mathrm{r}}$ is the upper support region threshold of the restricted $\log \mathrm{Q}$.

Note that G.711 quantizer is standardized and its parameters are known in advance $(\mu=255, N=256)$. Hence, for a given $M$ the proposed quantizer will be completely defined by the parameters $\mu^{\mathrm{r}}$ and $x_{\max }^{\mathrm{r}}$ of the restricted $\log \mathrm{Q}$. These parameters are optimally determined, where minimal distortion is used as a criterion

$$
\frac{\partial D_{t}}{\partial \mu^{\mathrm{r}}}=0, \quad \frac{\partial D_{t}}{\partial x_{\max }^{\mathrm{r}}}=0
$$

Let us define the SQNR and bit rate $R$ of the proposed dual-mode quasi-logarithmic quantizer

$$
\mathrm{SQNR}=10 \log _{10} \frac{1}{D_{t}}, \quad R=\log _{2} N+\frac{1}{M} .
$$

where the first term in (28) denotes the number of bits per sample required for quantization using $N$ levels and the second term is the side information that carries the information about the selected quantizer for a particular frame.

\section{Numerical results and discussion}

In this section, we present the performance assessment of the proposed combined quasi-logarithmic quantizer with embedded G.711 codec for zero-mean and unit variance Laplacian sources. The total distortion, as defined in (24), depends on the parameters $M, \mu^{\mathrm{r}}$ and $x_{\max }^{\mathrm{r}}$. Accordingly, for each frame length $M$ we established the ranges of $\mu^{\mathrm{r}} \in[2,5]$ and $x_{\max }^{\mathrm{r}} \in[2,5]$ where their optimal values are searched for in order to satisfy (26).

Figure 2 plots the dependence of SQNR on the frame length $M$ for the proposed quantizer designed for $N=$ 256 quantization levels, for the case when the corresponding parameters $\mu^{\mathrm{r}}$ and $x_{\max }^{\mathrm{r}}$ are optimally (according to (26)) and non-optimally chosen (not satisfying (26)). As it is evident, the SQNR decreases as $M$ increases and non-optimal selection degrades performance.

Furthermore, we analyze the selection of the optimal frame size $M$. In contrast to [13-15] where the special criterion is used to choose the appropriate $M$, in this 


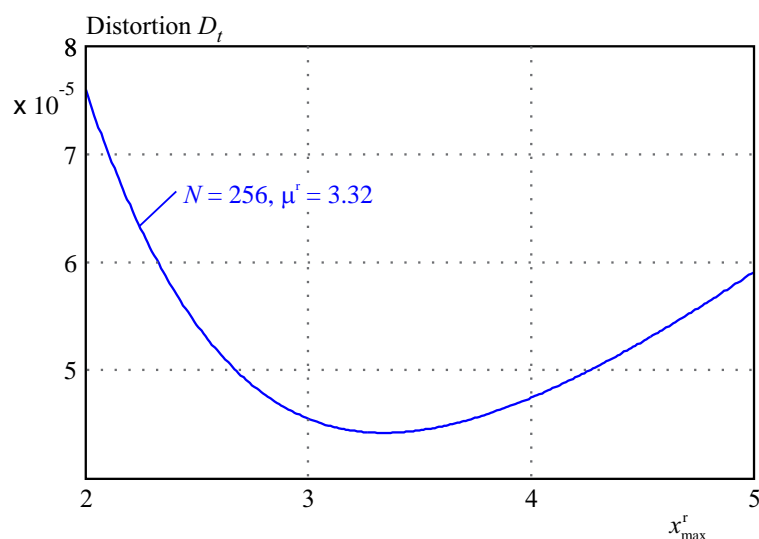

Fig. 4. Selection of the optimal value of $x_{\max }^{\mathrm{r}}$ and $\mu^{\mathrm{r}}$

paper we conduct a different procedure using the maximal SQNR as a criterion.

Let us first define

$$
\mathrm{SQNR}^{\mathrm{cor}}=\mathrm{SQNR}^{\mathrm{PROP}}-\frac{6 \mathrm{~dB}}{M} .
$$

Note that the proposed quantizer has the bit rate increased by one bit per frame compared to G.711 codec, see (28). This is the side information that carries the information about the selected quantizer in the dual-mode quasi-logarithmic quantizer for a particular frame. The second term in (29) is subtracted only to determine the equivalent SQNR that would be obtained for the same bit rate as in G.711, iedecreased by $6 \mathrm{~dB} /$ bit [1]

$\mathrm{SQNR}^{\text {cor }}$ as a function of the frame size $M$ is depicted in Fig. 3. It can be observed that the proposed quantizer significantly outperforms the G.711 quantizer [6] for the same bit rate ( $R=8 \mathrm{bit} / \mathrm{sample}$ ) in the entire range of $M$. The maximum improvement in SQNR is achieved for $M=7$ and amounts $5.41 \mathrm{~dB}$. For that case, the corresponding values of the compression factor and upper support region threshold that minimize distortion $D_{t}$ are $\mu^{\mathrm{r}}=3.32$ and $x_{\max }^{\mathrm{r}}=3.34$, as Fig. 4 shows. Now, by adopting the above ascertained parameters the proposed quantizer is completely defined. Note that it is possible to encode the input samples with nearly 0.9 bit less than G.711 quantizer, simultaneously providing the same signal quality measured as SQNR ( $6 \mathrm{~dB} /$ bit rule).

In Fig. 5 we present SQNR vs bit rate for the proposed dual-mode quasi-logarithmic quantizer and the different baselines including the optimal compandor [18], the piecewise optimal compandor [10] and the uniform quantizer [5]. Granular and overload distortion of an optimal compandor are defined as follows [18]:

$$
\begin{aligned}
& D_{\mathrm{g}}=\frac{9}{2 N^{2}}, \\
& D_{\mathrm{o}}=\frac{1}{2} \exp \left(-\sqrt{2} x_{\max }\right) .
\end{aligned}
$$

The results are provided for the number of quantization levels equal to 128,256 and 512, which for the baselines corresponds to bit rates 7,8 and 9 bits/sample, respectively. Note that we use optimal parameters for $N=128$

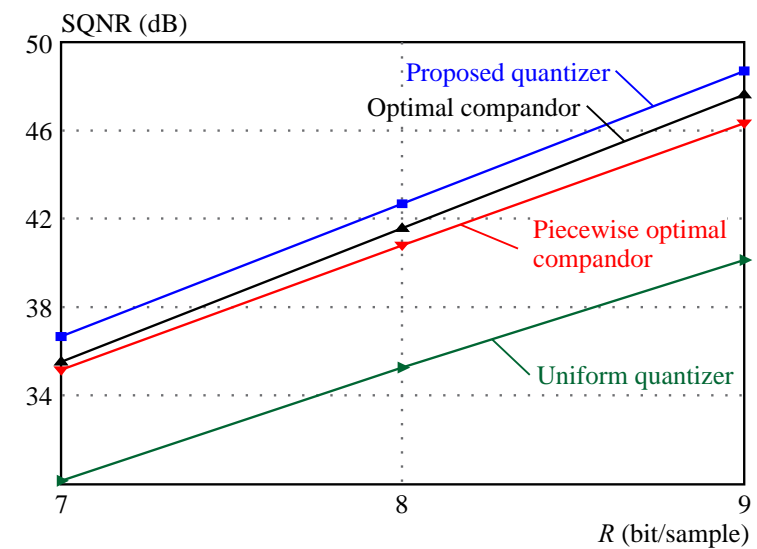

Fig. 5. SQNR vs bit rater

$\left(\mu^{\mathrm{r}}=3.32\right.$ and $\left.x_{\max }^{\mathrm{r}}=3.34\right)$ and $N=256\left(\mu^{\mathrm{r}}=3.34\right.$ and $\left.x_{\max }^{\mathrm{r}}=3.35\right)$. We report gain in SQNR of about $1.08 \mathrm{~dB}$ to $1.16 \mathrm{~dB}$ compared to the optimal compandor given in [18], $1.52 \mathrm{~dB}$ to $2.36 \mathrm{~dB}$ compared to the piecewise linear optimal compandor given in [10], and $6.54 \mathrm{~dB}$ to $8.25 \mathrm{~dB}$ compared to the uniform quantizer given in [5]. The obtained gain in SQNR clearly indicates that the proposed quantizer is an efficient solution for high-quality quantization.

\section{Conclusion}

The dual-mode quasi-logarithmic quantizer designed for the memoryless Laplacian source is proposed in this paper. It uses the restricted logarithmic and the unrestricted embedded G.711 quantizer having the same number of quantization levels. The restricted logarithmic quantizer is used for all frames having the maximal amplitude within its support region, while for other ones the unrestricted embedded G.711 quantizer is employed. The gain in SQNR of $5.41 \mathrm{~dB}$ over the standardized G.711 quantizer is reported, justifying the motivation for introduction of the proposed quantizer. Although our quantizer slightly increases the design complexity, it can be traded for the achieved gain. The proposed quantizer can be successfully employed in all applications where the high-quality quantization of signals following the Laplacian PDF is of primary interest.

\section{Acknowledgements}

This work was partly funded by the Ministry of Education and Science of the Republic of Serbia, grant no. TR32051 and TR32035, within the Technological Development Program, and grant no. SK-SRB-2016-0030, within the bilateral program with the Slovak Research and Development Agency.

\section{REFERENCES}

[1] N. S. Jayant and P. NolL, "Digital Coding of Waveforms", Principles and Applications to Speech and Video, New Jersey: Prentice Hall, 1984. 
[2] L. Hanzo, C. Somerville and J. Woodard, "Voice and Audio Compression for Wireless Communications", John Wiley \& Sons - IEEE Press, 2007.

[3] A. Gersho and R. M. Gray, "Vector Quantization and Signal Compression", Kluwer Academic Publishers, 1992.

[4] C. Chu, "Speech Coding Algorithms", New Jersey: John Wiley \& Sons, 2005.

[5] D. Hui and D. L. Neuhoff, "Asymptotic Analysis of Optimal Fixed-Rate Uniform Scalar Quantization", IEEE Trans. Inf. Theory, vol. 47, no. 3, pp. 957-977, 2001.DOI: 10.1109/18.915652.

[6] "International Telecommunication Union. ITU-T G. 711: Pulse code modulation of voice frequencies", 1972.

[7] Z. Peric, L. Velimirovic and M. Dincic, "Improved Linearization of the Optimal Compression Function for Laplacian Source", Journal of Electrical Engineering, vol. 65, no. 3, pp. 179-183, 2013.DOI: $10.2478 /$ jee-2014-0028.

[8] R. D. Shah and S. K. Singh, "Media Access Delay and Throughput Analysis of Voice Codec with Silence Suppression on Wireless Ad Hoc Network", Proc. of 7th International Conference on Communication, Computing and Virtualization, vol. 79, pp. 940-947, 2016.DOI: 10.1016/j.procs.2016.03.119.

[9] E. Antwi-Boasiako, E. Kuada and K. Boakye-Boateng, "Role of Codec Selection on the Performance of IPsec Secured VoIP", Proc. of IEEE International Conference on Advances Computing, Communications and Informatics (ICACCI), 2016.

[10] L. Velimirovic and S. Maric, "New Adaptive Compandor for LTE Signal Compression based on Spline Approximations", ETRI Journal, vol. 38, no. 3, pp. 463-468, 2016, DOI: 10.4218/etrij. 16.0115.0506.

[11] M. Dincic and Z. Peric, "Log-Polar Quantizer with the Embedded ITU-T G. 711 Codec", Radioengineering, vol. 19, no.4, pp. $712-717,2010$.

[12] S. Na, "On the Support of Fixed-Rate Minimum Mean-Squared Error Scalar Quantizers for a Laplacian Source", IEEE Transactions on Information Theory, vol. 50, no. 5, pp. 937-944, 2004.DOI: 10.1109/TIT.2004.826686.

[13] Z. Peric and J. Nikolic, "An Adaptive Waveform Coding Algorithm and its Application Speech Coding", Digital Signal Processing, vol. 22, no. 1, pp. 199-209, 2012, DOI: $10.1016 /$ j.dsp.2011.09.001.

[14] Z. Peric and J. Nikolic, "High-Quality Laplacian Source Quantisation using a Combination of Restricted and Unrestricted Logarithmic Quantisers", IET Signal Processing, vol. 6, no. 7, pp. 633-640, 2012, DOI: 10.1049/iet-spr.2011.0213.

[15] J. Nikolic, Z. Peric and A. Jovanovic, "Two Forward Adaptive Dual-Mode Companding Scalar Quantizers for Gaussian Source", Signal Processing, vol. 120, no. C, pp. 129-140, 2016, DOI: $10.1016 /$ j.sigpro.2015.08.016.

[16] Z. Peric, M. Dincic, D. Denic and A. Jocic, "Forward Adaptive Logarithmic Quantizer with New Lossless Coding Method for Laplacian Source", Wireless Personal Communications, vol. 59, no. 4, pp. 625-641, 2011, DOI: 10.1007/s11277-010-9929-3.

[17] D. Aleksic, Z. Peric and J. Nikolic, "Support Region Determination of the Quasilogarithmic Quantizer for Laplacian Source", Przeglad Elektrotechniczny, vol. 88, no. 7, pp. 130-132, 2012.

[18] S. Na, "Asymptotic Formulas for Mismatched Fixed-Rate Minimum MSE Laplacian Quantizers", IEEE Signal Processing Letters, vol. 15, pp. 13-16, 2008, DOI: 10.1109/LSP.2007.910240.

Received 24 May 2017

Bojan D. Denić was born in Vrbestica, township Urosevac, Serbia, in 1986. He received BSc and MSc degrees degree in electronics and telecoq mmunications from the Faculty of Technical Sciences, University of Pristina, Serbia. His current research interests include scalar quantization and signal processing. He is currently a $\mathrm{PhD}$ candidate at the Faculty of Electrical Engineering, University of Niš, Serbia and a scholar of Serbian Ministry of Education, Science and Technological Development.

Zoran H. Perić was born in Niš, Serbia, in 1964. He received the BSc degree in electronics and telecommunications from the Faculty of Electronic Engineering, University of Niš, Serbia, Yugoslavia, in 1989, and MSc degree in telecommunications from the University of Niš, in 1994. He received the $\mathrm{PhD}$ degree from the University of Niš, also, in 1999. He is currently Full Professor at the Department of Telecommunications, Faculty of Electronic Engineering, University of Niš, Serbia. His current research interests include the information theory, source and channel coding and signal processing. He is particularly working on scalar and vector quantization techniques in speech and image coding. He was author and coauthor in over 200 papers in digital communications. He has been a reviewer for IEEE Transactions on Information Theory, IEEE Transactions on Signal Processing, IEEE Transactions on Communications, Compel, Informatica, Information Technology and Control, Expert Systems with Applications and Digital Signal Processing.

Vladimir Despotović received his diploma in engineering degree in telecommunications from the University of Niš, Faculty of Electronic Engineering, Serbia in 2003. He received his $\mathrm{PhD}$ degree in electrical engineering and computer science from the University of Niš in 2012. Currently he is working as Assistant Professor at the University of Belgrade, Technical Faculty of Bor, Serbia. Previously he was engaged as postdoctoral researcher at the University of Paderborn, Department of Communications Engineering, Germany. His main research interests include statistical signal processing, spoken language understanding, natural language processing, fractional calculus and machine learning. He is an author of 10 journal papers, one book chapter, one patent and over 25 conference papers.

Nikola Vučić was born in Niš, Serbia, in 1990. He graduated on the Faculty of Electronic Engineering, University of Niš, in 2014, obtaining the MSc degree for Electrical Engineering and Computer Science at the field of Telecommunications. His current research interests include the signal processing. He is currently a PhD candidate at the Faculty of Electronic Engineering, University of Niš, Serbia and a scholarship of the Serbian Ministry of Education, Science and Technological Development.

Predrag Petrović is the full member of Serbian Academy of Engineering Sciences. He got The Institute of Electrical and Electronics Engineers (IEEE) Vehicular Technology Society 1982 Runner-Up Paper of the Year Award in Communications Technology for the paper entitled Digitized speech transmission at VHF using existing FM mobile radios. His doctoral dissertation at the Faculty of Electrical Engineering of University of Beograd was awarded in 1983. In addition, he received the Year Award of Elektronska industrija in 1988 for the scientific contribution in the field of real-time automatic radio signals classification. He published 124 papers in international and national journals and conference proceedings. His R\&D work comprises 77 realized radio, EW, and radar systems and devices as well as 54 projects in telecommunication, mobile radio communications, software defined radio, broadcasting, information security. 